\author{
METEOROLOGY OF THE OUTER PLANETS \\ Garry E. Hunt \\ Laboratory for Planetary Atmospheres \\ Department of Physics and Astronomy \\ University College London \\ London WC1E 6BT
}

\title{
INTRODUCTION
}

One of the fundamental problems in atmospheric physics is concerned with providing improved weather forecasts for the Earth and predicting the future climate of our planet. However, this is a particularly difficult task since the meteorology of the Earth is affected by a wide range of physical factors - clouds, oceans, continents, polar caps, deserts and forests - all of which respond differently to the solar energy which drives the weather systems. In addition, external factors, such as the changing energy from the sun, are thought to strongly influence the Earth's meteorology. To provide a broader understanding of atmospheric phenomena, detailed studies of planetary atmospheres have been conducted, since they provide a unique opportunity to investigate these basic problems in fluid dynamics under boundary conditions which are quite different from those found on the Earth.

The giant outer planets provide, at first sight, a more varied set of objects, since they are huge, rapidly rotating, low density planets with optically reducing atmospheres (Be1ton (1981), Goody (1981), Kondratyev and Hunt (1981)). The giant planets Jupiter, Saturn and Neptune possess internal heat sources (MacFarlane and Hubbard (1981)) while the present observations of Uranus suggest that it may be in radiative equilibrium. The effects of internal heating as an additional driving mechanism to differential solar heating for a planetary atmosphere are not fully understood.

In order to provide insight into this fundamental problem, we discuss in this paper the meteorologies of the atmospheres of the major planets. The detailed observations of Jupiter and Saturn by the Pioneer 11 and Voyager spacecraft now make it possible to provide quantitative discussions of the motions of these planetary 
atmospheres (see, for example, Hunt (1981)). We therefore first review our basic knowledge of Jupiter and Saturn and then discuss our current understanding of Uranus and Neptune.

\section{THE METEOROLOGIES OF JUPITER AND SATURN}

For more than 300 years, observations of large-scale cloud features have provided the basic information on the gross characteristics of the atmospheres of Jupiter and Saturn (see, for example, Peek (1958), Alexander (1962) and Smith and Hunt (1976)). The visible appearance of Jupiter is one of alternating cloud bands of differing colours separated by jet streams. Superimposed upon these cloud systems are large-scale features, such as the Great Red Spot and the three white ovals, which appear to have lifetimes varying from decades to centuries (Smith et al $(1979 a, b)$ ). Saturn is in some ways similar to Jupiter. Although the banded structure is clearly seen, there is a marked hemispherical asymmetry in the clarity of the visible features due to possible seasonal changes in the upper atmospheric haze layers. Furthermore, there are considerably less large-scale cloud features to be seen in the Saturnian atmosphere (Smith et al (1981)).

Unlike the meteorological systems of the terrestrial planets, the weather systems of these planets are not solely driven by differential solar heating, since they both possess strong internal heat sources. Jupiter emits $1.67 \pm 0.13$ times the energy it receives from the sun, while the equivalent factor for Saturn is $22.8 \pm 0.9$. Consequently, the meteorologies of these planets are influenced by the two energy sources and by the strong rotation. All the cloud velocities on Jupiter are referenced to the System III period of $9^{\mathrm{h}_{5}} 5^{\mathrm{m}} 29.711^{\mathrm{s}}$ and on Saturn to the System III period of $10^{\mathrm{h}} 39.9 \pm 0.5^{\mathrm{m}}$.

On a large scale, there is little, if any, pole to equator energy transfer at the level of the visible clouds, which is a major difference between the Earth and the giant planets. The Pioneer 11 measurements of Ingersoll et al (1976) have shown that on Jupiter the difference between the equator and pole temperatures is no more than $3 \mathrm{~K}$. The belt/zone structure terminates at $\pm 45^{\circ}$. 
Although Saturn possesses similarly small temperature gradients, at the time of the Voyager encounter the temperatures at the $209 \mathrm{mb}$ level were noticeably warmer in the southern, compared with the northern, hemisphere of the planet (Hanel et a1 (1981)). This is a seasonal effect caused by Saturn's $27^{\circ}$ obliquity. The radiative time constant at this atmospheric level is approximately equal to the Saturnian year (Cess and Caldwell (1979)), so that the thermal response is expected to $\mathrm{lag}$ behind the seasonal forcing by about a quarter of the seasonal cycle, resulting in the observed cold northern hemisphere. On Saturn, the light and dark cloud bands also appear to extend to latitudes of about $70^{\circ}$ in each hemisphere.

ZONAL VELOCITY vS LATITUDE ON SATURN and JUPITER

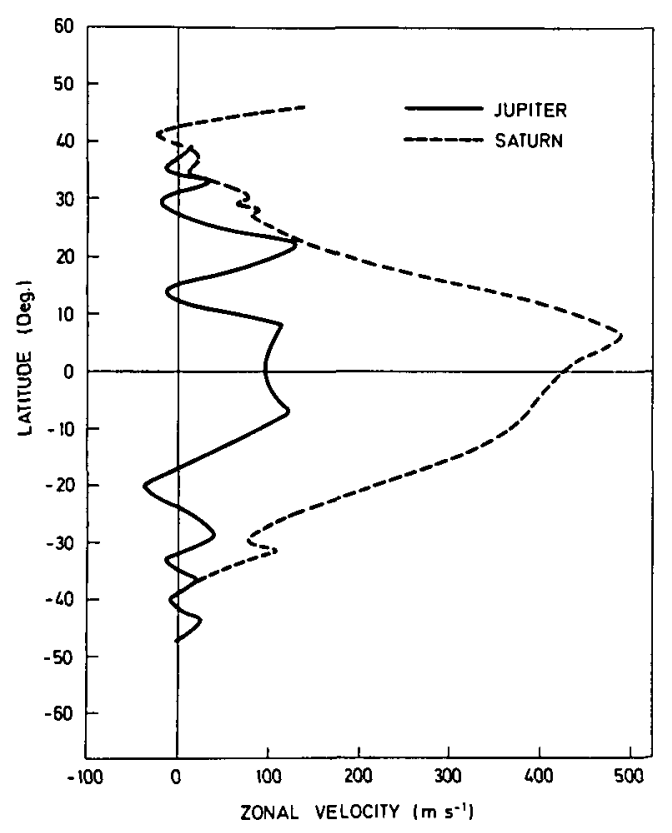

Fig 1 - Comparison of the zonal velocity of Jupiter (Voyager 1; February 1979)

with that of Saturn (Voyager 1, November 1980).
In figure 1, we have compared the zonal velocities of Jupiter and Saturn, obtained by tracking cloud elements between specific frames of Voyager images. The most noticeable differences between these profiles are the presence of the broad, strong equatorial jets in the atmosphere of Saturn, and the virtual

absence of easterly jets. Retrograde jets have been found

in the Saturn atmosphere at planetocentric latitudes of $\sim 41,58,78^{\circ} \mathrm{N}$ 
and 41 and $58^{\circ} \mathrm{S}$. These major differences between the zonal wind profiles must reflect differences in the internal structure and driving mechanisms of these atmospheres.

Studies of the eddy momentum flux by Beebe et al (1980), Ingersoll et al (1980) and Hunt (1981) have provided some insight into possible driving mechanisms which are summarised in figure 2. Available potential energy for the atmosphere is generated by the buoyancy of the cloud systems, which is transferred into kinetic energy by the planetary rotations. However, we find for Jupiter that the main motions are being driven by the conversion of eddy

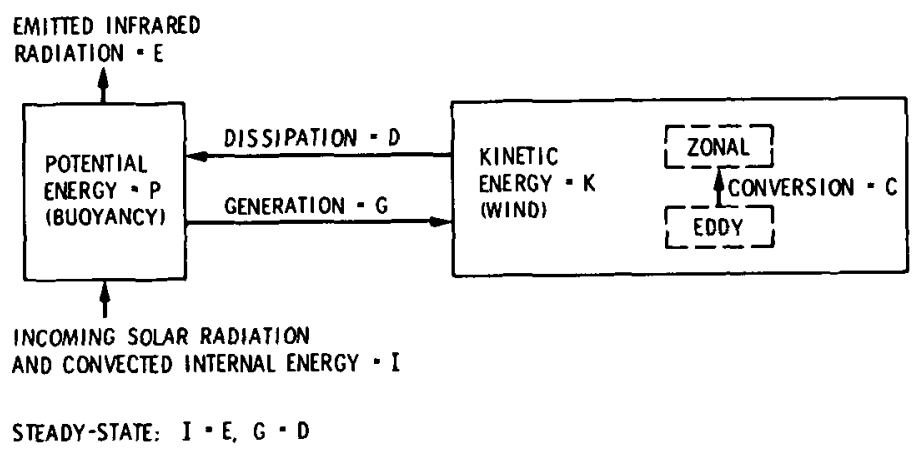

Fig 2 - A schematic representation of the energy balance mechanisms for the generation of the motions in planetary atmospheres

kinetic energy into zonal mean kinetic energy as in the Earth's atmosphere (Holton (1972)). The rate of conversion of eddy kinetic energy into mean kinetic energy is in the range 1.5 to $3 \mathrm{~W} . \mathrm{m}^{2}$ for a layer 2.5 bars deep. The time constant for resupply of zonal mean kinetic energy by the eddies is in the range of 2-4 months, which is less than the interval between the Voyager encounters. Clearly, this situation cannot proceed unbounded, so that some larger atmospheric system must therefore owe its existence to the extraction of eddy momentum from the flow in which it is embedded. It is possible that the Great Red Spot and the white ovals behave in this way. The zonal wind profiles shown in figure 1 illustrate 
essentially no correlation with the banded structure of either Jupiter or Saturn (Ingersoll et al (1980), Hunt (1981), Smith et al (1981)). This suggests that the visible clouds are controlled by the radiative/dynamical properties within the upper troposphere and clouds rather than in the deep atmosphere associated with the jets. The rate of conversion is more than $10 \%$ of the total infrared heat flux for Jupiter in contrast to $0.1 \%$ for the Earth. This 100 fold difference suggests that the thermomechanical energy cycles are very different on the two planets. It is certainly possible that the zonal flux extends much deeper than the eddies and therefore is affected on a timescale much longer than four months. The stability of the Jovian (and Saturnian) jets over time periods of centuries, which is in complete contrast to the rapidly varying visible appearance of the planet, is consistent with the assumption that the jets are associated with the deeper layers of the atmosphere.

To explain the differences in the zonal profiles of the planets shown in figure 1 , we note that for Jupiter internal heating supplies less energy to the atmosphere than solar heating. For Saturn, however, internal heating supplies roughly twice the energy than is received from solar heating. This internal heating would tend to increase the relative importance of small scale convection on Saturn. In addition, Saturn has a lower value for gravity, which leads to a larger atmospheric scale height, and a corresponding larger scale of convective motions. This would then tend to increase the amount of available potential energy of the system, which, on a rapidly rotating planet, would produce the observed stronger motions (figures 1 and 2 ). It is apparent therefore that the energy cycle for these planets provides the basic information to explain the observed flows, which should be taken into account when we now address Uranus and Neptune, where considerably less observational information is available.

\section{THE METEOROLOGIES OF URANUS AND NEPTUNE}

Uranus is the first planet so far from Earth that dynamical activity, manifested by clouds, cannot be easily observed by ground 
based observations. Consequently, we have no direct knowledge about atmospheric motions. Our knowledge about the structure of the cloud layers, which would act as a tracer of any motions, is itself very limited. While methane cloud layers could form in the atmosphere (Be1ton (1981)), it is also possible that any structure within this cloud would be obscured from view by extensive aerosol layers. At deeper levels, layers of $\mathrm{NH}_{3}$ cloud and $\mathrm{NH}_{4} \mathrm{SH}$ clouds are expected. The observed changes in the microwave spectrum of Uranus by Klein and Turegano (1978) are possibly due to variations in the structure of these deep clouds.

Both Uranus and Neptune show long term brightness changes in the visible portion of the spectrum. Lockwood and Thompson (1978) have found a $1 \%$ increase in the absorption in the 6190 and $7261 \AA$ $\mathrm{CH}_{4}$ bands over a two month interval. Observations from 1953 to 1965 show that the B magnitude of Neptune increased from 0.02 mag., while that for Uranus decreased from $0.04 \mathrm{mag}$. Then from 1972 to 1976, Neptune brightened by $0.007 \mathrm{mag} /$ year, while the brightness of Uranus changed by $0.012 \mathrm{mag} /$ year. These changes are certainly the result of variations in the solar $\mathrm{UV}$ flux, which then alters the albedo of the planet through the corresponding photochemical and constituent changes to the upper atmosphere and clouds. This suggests that the radiative budget of these planets, which must be important in the general circulation of these planetary atmospheres, will possess some significant variations, and thus influence the motions.

While the images of Jupiter and Saturn show definite detail of the light/dark cloud structures, there is little to suggest that Uranus and Neptune have a banded appearance. Alexander (1965) suggested that when the equatorial regions of the planet are visible and the viewing excellent two faint belts on either side of a bright zone are sometimes seen on Uranus, using larger telescopes. Antoniadi thought that when he observed the planet, the belts appeared exactly parallel to the general plane of the satellite orbits and not tilted at about $20^{\circ}$ as other observers had reported. Dollfus (1970) remarked that the features he saw did not seem to be belts. The best image currently available, taken at $890 \mathrm{~nm}$, shows 
evidence of the bright limb of Uranus, which is suggestive of a haze layer high in the atmosphere (see Smith (1981)). Neptune has been more difficult to observe, and the observations by Smith (1981) show possible evidence of cloud structure with a dark equatorial belt separating brighter regions in each hemisphere.

It is necessary to examine the basic atmospheric properties of the planets, which are summarised in Table 1.

TABLE 1: Basic Properties of Planetary Atmospheres

\begin{tabular}{|c|c|c|c|c|c|}
\hline & Albedo & $\begin{array}{l}\text { Effective } \\
\left.\text { Temp ( }{ }^{\circ} \mathrm{K}\right)\end{array}$ & $\begin{array}{l}\text { Measured } \\
\text { Temp }\left({ }^{\circ} \mathrm{K}\right)\end{array}$ & $\begin{array}{c}\text { Adiabatic } \\
\text { Lapse Rate } \\
\left({ }^{\circ} \mathrm{K} / \mathrm{km}\right)\end{array}$ & $\begin{array}{l}\text { Radiative } \\
\text { Time } \\
\text { Constant }\end{array}$ \\
\hline Earth & 0.30 & 256 & 256 & थ9.8 & 60 days \\
\hline Jupiter & $0.33 \pm 0.02$ & 106 & $124.9 \pm 0.3$ & 1.9 & ح6 yrs \\
\hline Saturn & $\sim 0.36$ & $76 \pm 4$ & $96.5 \pm 2.5$ & 0.9 & ح6 yrs \\
\hline Uranus & $0.34-0.5$ & $55-58$ & $58 \pm 2$ & $\sim 1.0$ & $\sim 600$ yrs \\
\hline Neptune & $0.34-0.5$ & $43-46$ & $\sim 55$ & $\sim 1.4$ & $\sim 2200 \mathrm{yrs}$ \\
\hline
\end{tabular}

These tabulations indicate that, unlike the other major planets, Uranus does not appear to possess an internal heat source to provide an additional driving mechanism for the motions. The long radiative time constant compared with the length of the Uranian day (Houghton, 1977; Goody, 1981) would suggest a relatively more stable atmosphere than the other planetary atmospheres. Stone (1973, 1975) suggested that the Richardson number may be as large as 2800 , confirming this interpretation.

Uranus does, however, possess some important differences.from the other planets. The large inclination of the axis of rotation will mean that each hemisphere will spend a substantial period without sunlight once each Uranian year. At the present time, the north pole of the planet is just turning towards the Earth after having turned away from the sun for more than 40 years. The atmosphere will then cool substantially in the absence of meridional heat transport.

Although Uranus has a rapid rotation period of $16.31 \pm 0.27$ hrs 
(Goody (1981)), the large inclination of the equator means that during the course of one orbit all equatorial regions receive less heat from the sun than the polar latitudes. Consequently, the poles will be hotter than the equator, so that from the thermal wind equation the mean zonal winds will be easterly, in contrast to the mean westerly winds of Jupiter and Saturn.

Even in the absence of any major internal heating, it is natural to expect that on a rapidly rotating planet certain natural horizontal scales of motion will be generated. We may anticipate barotropic instabilities of $\sim 1000 \mathrm{~km}$ and baroclinic instabilities of $\sim 600 \mathrm{~km}$ for a mean velocity of $\sim 2 \mathrm{~ms}^{-1}$. The corresponding time scales of these features would be about 40 days and 8 days respectively. We would not necessarily expect to find large scale convective motions in the atmosphere of Uranus. As a consequence, we may expect the motions to be far less turbulent than on Jupiter and Saturn, and possibly even the Earth.

Neptune may, however, have large scale motions. Joyce et al (1977) have observed a factor of four change in the reflectance of the planet between 1 and $4 \mu \mathrm{m}$ during the period 1975-76, while the simultaneous measurements of Uranus showed a negligible change. More recently, Cruikshank (private communication (1981)) has reported similar rapid changes in the structure of Neptune. Pilcher (1977) suggested that these changes could be accounted for by large scale variations in the cloud structure. Since the observations refer to the whole planetary disk, we may anticipate considerable structure in the cloud layers when higher spatial resolution observations are made later this decade. Certainly, it is possible that Neptune could have strong motions. Belton et al (1981) believe that the simultaneous presence of three periodicities of 17.73, 18.56 and 19.29 hours for the rotational period of the planet, together with related harmonics in the $\mathrm{J}-\mathrm{K}$ colour, is consistent with zonal winds of up to $109 \mathrm{~ms}^{-1}$. Furthermore, the internal heating would be dominant over the available absorbed solar energy and therefore by the energy budgets of figure 2, leading to strong motions. 


\section{CONCLUSIONS}

The meteorology of Uranus may be quite different from the motions of the other large planets. The apparent absence of internal heating and the large obliquity of the axis of rotation on this rapidly rotating planet make Uranus quite different from its planetary neighbours. We anticipate only weak motions, generated primarily by the differential solar heating, which, at the time of the Voyager encounter in 1986, will be a maximum in the polar region. The meteorology of Uranus provides a major test of our understanding of planetary weather systems, and must be monitored by Space Telescope during the next decade to extend the high spatial Voyager observations.

\section{ACKNOWLEDGEMENTS}

This work is supported by the Science and Engineering Research Council. Contribution 97 of the Laboratory for Planetary Atmospheres.

\section{REFERENCES}

Alexander, A.F.O'D (1962) The Planet Saturn (Faber and Faber)

Alexander, A.F.O'D (1965) The Planet Uranus (Faber and Faber)

Beebe, R.F., Ingersoll, A.P., Hunt, G.E., Mitche11, J.L. and Muller, $\mathrm{J}-\mathrm{P}$ (1980) Measurements of Wind Vectors, Eddy Momentum Transports and Energy Conversions in Jupiter's Atmosphere from Voyager 1 Images. Geophys. Res. Letts. $7,1-4$

Belton, M.J.S. (1981) An Introductory Review of our Present Understanding of the Structure and Composition of Uranus' Atmosphere (this volume)

Belton, M.J.S., Wallace, L. and Howard, S. (1981) The Periods of Neptune; Evidence for Atmospheric Motions. Icarus (in press)

Cess, R.D. and Caldwe11, J. (1979) A Saturnian Stratospheric Seasonal Climate Model. Icarus 38, 349-357

Dollfus, A. (1970) New Optical Measurements of the Diameters of Jupiter, Saturn, Uranus and Neptune. Icarus 12, 101-117

Goody, R. (1981) The Rotation of Uranus (this volume)

Hanel, R. et al (1981) Infrared Observations of the Saturnian System from Voyager 1. Science 212, 192 
Holton, J. (1973) An Introduction to Dynamical Meteorology (Academic Press)

Houghton, J. (1977) The Physics of Atmospheres (CUP)

Hunt, G.E. (1981) The Atmospheres of Jupiter and Saturn. Phil. Trans. Roy. Soc. (in press)

Ingersol1, A.P., Munch, G., Neugebauer,G., Orton, G.S. (1976)

Results of the Infrared Radiometer Experiment on Pioneers 10 and 11 in Jupiter (ed.by T. Gehrels) p.197-205. University of Arizona Press

Ingersoll, A.P., Beebe, R.F., Mitchell, J.L., Garneau, G., Yagi, G., Muller, J-P and Hunt, G.E. (1980) Interaction of Eddies and Mean Zonal Flow on Jupiter as Inferred from Voyager 1 and 2 Images. J. Geophys. Res. (in press)

Joyce, R.R., Pilcher, C.B., Cruikshank, D.P, and Morrison, D. (1977) Evidence for Weather on Neptune I. Astrophys. J. 214, 657662

Klein, M.J. and Turegano, J.A. (1978) Evidence of an Increase in the Microwave Brightness Temperatures of Uranus. Astrophys. J. 224, L31-34

Kondratyev, K.Ya and Hunt, G.E. (1981) Weather and Climate on Planets (Pergamon Press)

Lockwood, G.W. and Thompson, D.T. (1978) A Photometric Test of Rotational Periods for Uranus and Time Variations of Methane Band Strengths. Astrophys. J. 221, 689-693

MacFarlane, J.J. and Hubbard, W. (1981) Internal Structure of Uranus (this volume)

Peek, B. (1958) The Planet Jupiter (Faber and Faber)

Pilcher, C.B. (1977) Evidence for Weather on Jupiter II. Astrophys. J. 214, 663-666

Smith, B.A. et al (1979a) The Jupiter System through the Eyes of Voyager 1. Science 204, 951-972

Smith, B.A. et a1 (1979b) The Galilean Satellites and Jupiter : Voyager 2 Imaging Science Results. Science 206, 927-951

Smith, B.A. et al (1981) Encounter with Saturn:Voyager I Imaging Science Results. Science 212, 163-191

Smith, B.A. (1981) Imaging Studies of the Outer Planets (this volume) 
Smith, B.A. and Hunt, G.E. (1976) Motions and Morphology of Clouds in the Atmosphere of Jupiter in Jupiter (ed.by T. Gehrels) (University of Arizona Press)

Stone, P. (1973) The Dynamics of the Atmospheres of the Major Planets. Space Sci. Rev. 14, 444-459

Stone, P. (1975) The Atmosphere of Uranus. Icarus 24, 292-298 